\title{
Arm Blanch Test-A Simple Sign for Dengue Diagnosis
}

\author{
Bethou Adhisivam ${ }^{1}$ (]) Venkatesh Chandrasekaran ${ }^{2}$
}

Received: 13 December 2021 / Accepted: 31 January 2022 / Published online: 8 March 2022

(c) Dr. K C Chaudhuri Foundation 2022

To the Editor: World Health Organization (WHO) has included the tourniquet test as a criterion for probable dengue, and a positive test reflects both capillary fragility and thrombocytopenia [1]. However, this test has a greater positivity rate in individuals with more severe forms of the disease but cannot exclude dengue infection [2-4]. Moreover, the test requires a sphygmomanometer and a time duration of at least $5 \mathrm{~min}$. We describe here a simpler alternative to the tourniquet test, the arm blanch test (ABT) which may be useful in making a clinical diagnosis of dengue early.

The physician should make a firm grip of the child's arm with his/her bare hand, sustain the pressure for $30 \mathrm{~s}$ and then release the grip. The skin surface of the arm beneath the physician's fingers will be blanched and with release of pressure, erythematous margins will be noted around the blanched areas. These children with a positive ABT developed other clinical features of dengue including skin and palmar erythema on follow-up. They also had hematological and serological investigations supporting the diagnosis of dengue. Normal fair-skinned children may have mild transient erythema with ABT. However, the erythema noted with children with dengue will be striking. As we blanch the skin, ABT can be useful even in children with a dark tan. ABT can be quicker than the tourniquet and can be a potential screening test even before the appearance other obvious signs of dengue. This clinical observation should be validated by a well-designed study. If we can do ABT and the tourniquet test in different arms of the same child with probable dengue, and correlate the clinical findings with serological parameters (in a good sample size), we can derive more meaningful conclusions.

\section{Declarations}

Conflict of Interest None.

\section{References}

1. Cao XT, Ngo TN, Wills B, et al. Evaluation of the World Health Organization standard tourniquet test and a modified tourniquet test in the diagnosis of dengue infection in Vietnam. Trop Med Int Health. 2002;7:125-32.

2. Kalayanarooj S, Vaughn DW, Nimmannitya S, et al. Early clini$\mathrm{cal}$ and laboratory indicators of acute dengue illness. J Infect Dis. 1997;176:313-21.

3. Kabra SK, Jain Y, Pandey RM, et al. Dengue haemorrhagic fever in children in the 1996 Delhi epidemic. Trans R Soc Trop Med Hyg. 1999;93:294-8.

4. Furlan NB, Tukasan C, Estofolete CF, Nogueira ML, da Silva NS. Low sensitivity of the tourniquet test for differential diagnosis of dengue: an analysis of 28,000 trials in patients. BMC Infect Dis. 2016;16:627.

Publisher's Note Springer Nature remains neutral with regard to jurisdictional claims in published maps and institutional affiliations.
Bethou Adhisivam

adhisivam1975@yahoo.co.uk

1 Department of Neonatology, Jawaharlal Institute of Postgraduate Medical Education and Research (JIPMER), Pondicherry 605 006, India

2 Department of Pediatrics, Jawaharlal Institute of Postgraduate Medical Education and Research (JIPMER), Pondicherry, India 